\title{
A simple manganous chloride and Congo red disc method for differentiating Neisseria gonorrhoeae from Neisseria meningitidis
}

\author{
T. O. ODUGBEMI, M. G. McENTEGART, AND S. HAFIZ \\ From the Department of Medical Microbiology, University of Sheffield Medical School, Beech Hill Road, \\ Sheffield S10 2RX, UK
}

SUMMARY Manganous chloride and Congo red incorporated into blotting paper discs have been used to differentiate gonococci from meningococci. The new technique is simple and reliable; the materials for the test are inexpensive. The method will increase the efficiency of distinguishing between the pathogenic Neisseria in any clinical bacteriology laboratory and especially in those in the tropical areas.

Clinically, the most important Neisseria are the gonococcus and the meningococcus. Gonorrhoea and other gonococcal infections are now the most common bacterial communicable diseases in the world. Meningococcal infections, although less widespread, cause pyogenic meningitis and meningococcal septicaemia, serious and often fatal conditions in many countries, especially in tropical areas. In recent times, gonococci have been isolated with increasing frequency from extragenital sites, such as the pharynx, and the clinical problems are further complicated by the isolation of meningococci from the genital tract (Catlin, 1973). Carbohydrate fermentation tests are commonly used to distinguish between these two pathogenic Neisseria, for the gonococcus produces acid from glucose only while the meningococcus produces acid from glucose and maltose. However, the results of fermentation patterns are not always easy to interpret and may fail to provide a clear identification in the routine clinical laboratories because of the failure of some strains of meningococci to produce detectable acid from maltose (Catlin, 1973). Although the immunofluorescence technique using specific antiserum has improved the means of differentiating gonococci from meningococci, there are still crossreactions between the two Neisseria (Reyn, 1965). Cox et al. (1977) recently described a radiometric technique to distinguish the two pathogenic Neisseria. This procedure is expensive, potentially hazardous, and beyond the reach of simple clinical bacteriology laboratories.

Received for publication 24 February 1978
In the course of our study on the effects of various cations on gonococci in vitro we noticed that $\vec{\varphi}$ whereas manganous chloride inhibited gonococci, even at a concentration of $10 \mu \mathrm{M}$, a meningococcus included among a series of controls was not inhibited even at a concentration of $10000 \mu \mathrm{M}$. This observation was investigated further as it seemed that it might provide a new, simple, and inexpensive test to distinguish gonococci from meningococci in any clinical bacteriology laboratory. In a preliminary survey of strains of gonococci, we found three strains that were relatively resistant to manganous chloride. In order to provide a more satisfactory method, we examined further compounds that show a differential toxicity for gonococci and meningococci, such as Congo red, recently found effective as a means of identifying penicillin-resistant, nonpenicillinase-producing gonococci (Payne and Finkelstein, 1977), ethylhydrocuprein hydrochloride (optochin), and bacitracin. We report here the results obtained with manganous chloride and Congo red mixture which was found most satisfactory for distinguishing gonococci from meningococci.

\section{Material and methods}

PREPARATION OF TEST DISCS

Manganous chloride (AnalaR), $0.5 \mathrm{~g}$, is dissolved in one litre of distilled water. Congo red (Koch-Light $\mathbb{D}$ Labs Ltd), $10 \mathrm{~g}$, is added to this solution (giving final concentrations of manganous chloride $0.05 \% \mathrm{w} / \mathrm{v}$ and Congo red $1 \% \mathrm{w} / \mathrm{v}$ ).

Whatman AA discs (diameter $13 \mathrm{~mm}$ ) are then $\frac{\rho}{\Phi}$ immersed in the solution, $7 \mathrm{ml}$ of the solution being 
sufficient to treat 50 discs. The wet impregnated discs are sterilised in a hot air oven at $160^{\circ} \mathrm{C}$ for 30 minutes and subsequently may be stored in sterile universal containers at $4^{\circ} \mathrm{C}$.

\section{CULTURES}

One hundred and six confirmed strains of Neisseria gonorrhoeae were tested, including 100 strains freshly isolated from patients attending the Special Clinic, Royal Infirmary, Sheffield and six known beta-lactamase producing strains (five strains supplied by Dr A. E. Jephcott and one strain supplied by Dr Turner, Public Health Laboratory, Liverpool). These were compared with 122 strains of meningococci representing mainly serogroups $A, B$, and $C$, supplied by Drs Abbott (Manchester), Fallon (Glasgow), and Young (Edinburgh). All cultures were first grown on Difco GC medium plus defined supplement (White and Kellogg, 1965) and checked for purity. Such fresh 18-24 hours cultures were then used to prepare a standard suspension in phosphatebuffered saline (after Brown's tube No. 1). For simplicity, a correct suspension can be obtained by emulsifying about 10 colonies of gonococci or meningococci in $2 \mathrm{ml}$ of phosphate-buffered saline; this produces a minimal turbidity. A 2-mm loopful of such bacterial suspensions was then inoculated onto each quarter of a $9-\mathrm{cm}$ plate of the same medium. The inoculum size is important. It is essential to have uniformly distributed and separated bacterial colonies because as in antibiotic testing large inocula producing confluent growth may make a sensitive organism appear resistant. The dry and sterile manganous chloride and Congo red discs were then placed in the centre of each inoculated quarter of the medium. The plates were incubated for 20 hours at $36^{\circ} \mathrm{C}$ in a humidified atmosphere of air and $10 \%$ carbon dioxide. Cultures showing a definite zone of inhibition around the edge of the discs were recorded as sensitive and identified as gonococci while those growing to the edge of the discs were recorded as resistant and identified as meningococci.

\section{Results}

The Figure shows a control strain of $N$. meningitidis resistant to the manganous chloride and Congo red disc and three strains of $N$. gonorrhoeae including F62, an international strain, $R 1$, a beta-lactamase producing strain, and AN97, a local strain, which is a penicillin-resistant, non-penicillinase producer; minimal inhibitory concentration (MIC) $1 \mathrm{mg} / \mathrm{l}$ with zones of inhibition around the discs. All the 106 strains of gonococci tested produced distinct zones of inhibition. Of 122 strains of meningococci tested, 121 were resistant to the manganous chloride and Congo red discs. The only sensitive strain of meningococcus was the NCTC 8554, an old laboratory

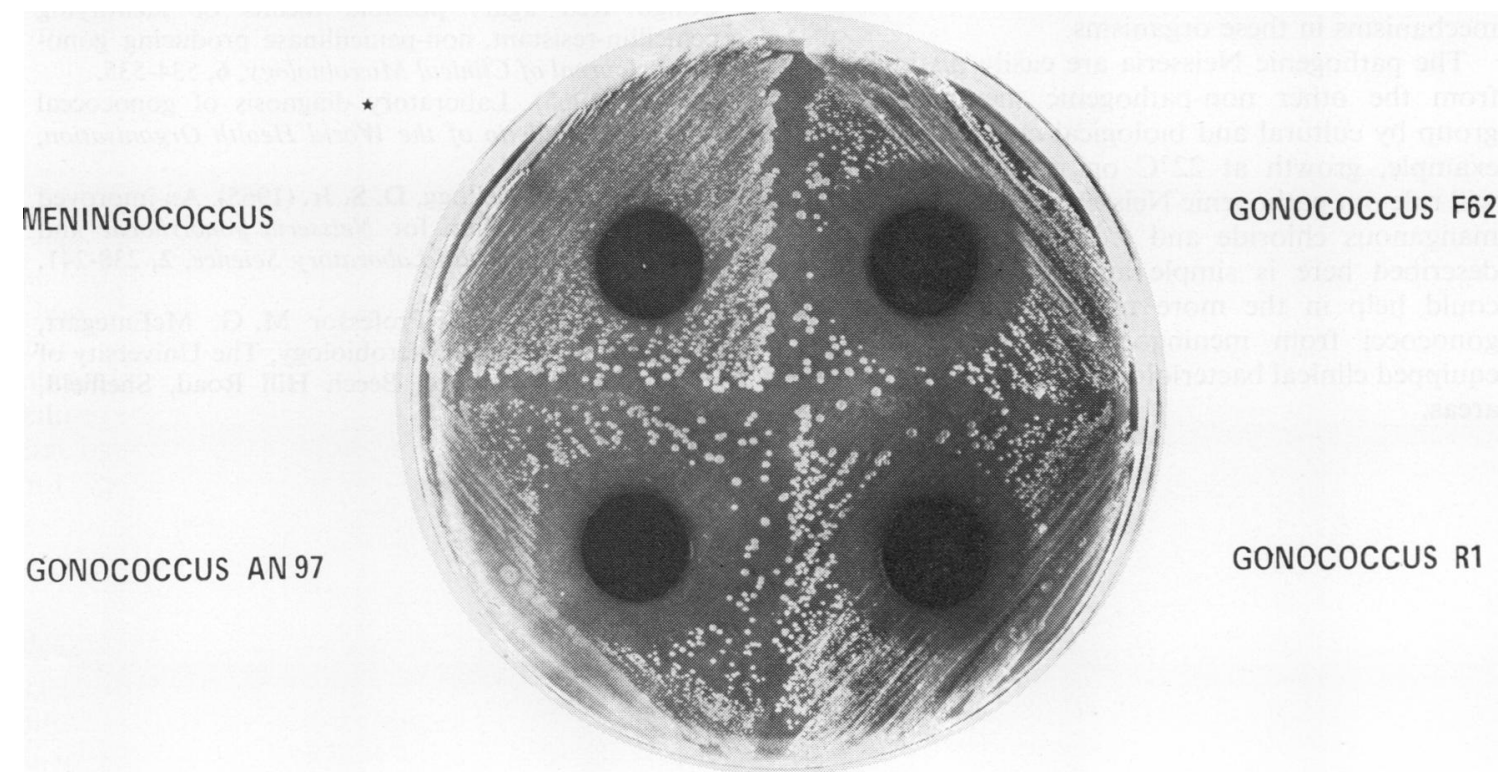

A control strain of $\mathrm{N}$. meningitidis resistant to the manganous chloride and Congo red disc and three strains of N. gonorrhoeae including strains F62, an international strain, R1, beta-lactamase producing strain, and AN97, a local penicillin-resistant, non-penicillinase producer with zones of inhibition around the discs. 
strain. The long storage in the laboratory could have been responsible for the sensitivity towards manganese; the remaining strains provide a more representative group of currently occurring strains for the United Kingdom and Nigeria. The maltose negative strains NCTC 8339 and N1032 (Dr Abbott) were correctly identified, as were three slow glucose fermenting strains $76 / 12561,77 / 02697$, and 77/19275 (Dr Fallon).

\section{Discussion}

Browning (1931), reviewing the metal-salt therapy, quoted the experimental results of Walbum, reported in 1921, in which manganese chloride was used successfully to treat mice injected with either tetanus spores, dysentery bacilli, or staphylococci. Although we know of no specific information about the differential toxicity of manganese salts for gonococci and meningococci, Firshein and Zimmerman (1964) found that manganese could depress the nucleic acid and protein syntheses of virulent strains of group A $\beta$-haemolytic streptococci while only nucleic acid synthesis was depressed in avirulent strains. Payne and Finkelstein (1977) suggested that Congo red might be acting on the gonococcal cell envelope. More fundamental research into the role of manganese, other trace elements, and dyes on the metabolism of pathogenic Neisseria may further increase our understanding of the pathogenicity mechanisms in these organisms.

The pathogenic Neisseria are easily distinguished from the other non-pathogenic members of the group by cultural and biological characteristics (for example, growth at $22^{\circ} \mathrm{C}$ on unenriched medium will rule out pathogenic Neisseria (Reyn, 1965)). The manganous chloride and Congo red disc method described here is simple and inexpensive, and it could help in the more precise differentiation of gonococci from meningococci even in sparsely equipped clinical bacteriology laboratories in remote areas.
We thank Drs R. J. Fallon, J. D. Abbott, and $H$. Young for supplying strains of meningococci, and Dr A. E. Jephcott for supplying beta-lactamase producing strains of gonococci. Dr Tolu Odugbemi is supported by a study leave grant from the University of Lagos.

\section{References}

Browning, C. H. (1931). In A System of Bacteriology in relation to Medicine (Medical Research Council), Vol. 6, edited by J. A. Arkwright et al., p. 527. HMSO, London. (Quoting Walbum, L. E. (1921). Action exercée par le chlorure de manganèse et d'autres sels métalliques sur la formation de l'antitoxine diphtérique et l'agglutinine de $B$. coli. Comptes Rendus des Séances de la Société de Biologie et de ses Filiates, 85, 761-764).

Catlin, B. W. (1973). Nutritional profiles of Neisseria gonorrhoeae, Neisseria meningitidis and Neisseria lactamica in chemically defined media and the use of growth requirements for gonococcal typing. Journal of Infectious Diseases, 128, 178-194.

Cox, F. R., Cox, M. E., Martin, J. R., and Stoer, U. H. (1977). Radiometric detection of carbohydrate catabolism by pathogenic neisseria. Journal of Clinical Microbiology, 6, 310-311.

Firshein, W., and Zimmerman, E. M. (1964). Differential toxicity of manganese for virulent and avirulent group A streptococci. Journal of General Microbiology, 36, 249-255.

Payne, S. M., and Finkelstein, R. A. (1977). Growth on Congo Red agar: possible means of identifying penicillin-resistant, non-penicillinase producing gonococci. Journal of Clinical Microbiology, 6, 534-535.

Reyn, A. (1965). Laboratory diagnosis of gonococcal infections. Bulletin of the World Health Organisation, 32, 449-469.

White, L. A., and Kellogg, D. S. Jr. (1965). An improved fermentation medium for Neisseria gonorrhoeae and other neisseria. Health Laboratory Science, 2, 238-241.

Requests for reprints to: Professor M. G. McEntegart, Department of Medical Microbiology, The University of Sheffield Medical School, Beech Hill Road, Sheffield, S10 2RX 